\title{
Switching at Low HIV-1 RNA into Fixed Dose Combinations: TDF/FTC/RPV is non-inferior to TDF/FTC/ EFV in first-line suppressed patients living with HIV
}

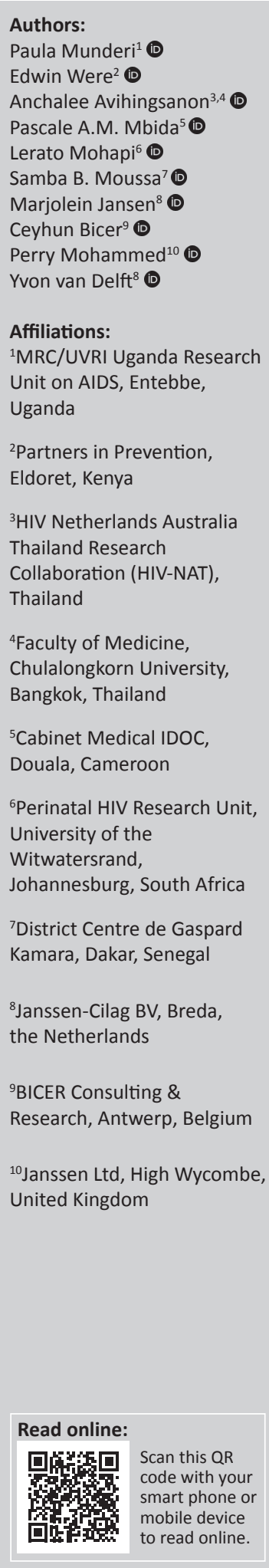

Background: In low- and middle-income countries (LMICs), a substantial unmet need for affordable single-tablet regimen (STR) options remains. Rilpivirine (RPV, TMC278) is formulated in a low-cost STR with tenofovir disoproxil fumarate (TDF) and emtricitabine (FTC).

Objectives: Switching at Low HIV-1 RNA into Fixed Dose Combinations (SALIF) compared RPV with efavirenz (EFV), both as STRs with TDF and FTC, in maintaining virologic suppression.

Methods: SALIF was a phase $3 b$, randomised, open-label, non-inferiority study in virologically suppressed adults (HIV-1 RNA $<50$ copies $/ \mathrm{mL}$ ) on non-nucleoside reverse transcriptase inhibitor (NNRTI)-based first-line antiretroviral therapy (ART) in Cameroon, Kenya, Senegal, South Africa, Uganda and Thailand. Patients $(N=426)$, stratified by NNRTI use, were randomised 1:1 to receive TDF/FTC/RPV (300/200/25 mg qd) or TDF/FTC/EFV (300/200/600 $\mathrm{mg}$ qd). Primary endpoint was proportion of patients with virologic suppression (HIV-1 RNA $<400$ copies $/ \mathrm{mL}$ ) at week 48 (intent-to-treat, modified Food and Drug Administration Snapshot, $10 \%$ non-inferiority margin).

Results: Patients received TDF/FTC/RPV $(n=213)$ or TDF/FTC/EFV $(n=211)$. At week 48, virologic suppression was maintained in 200/213 (93.9\%) patients in the RPV arm and 203/211 (96.2\%) in the EFV arm (difference $-2.3 \%$; 95\% confidence interval: $-6.4,+1.8$ ), demonstrating non-inferiority of TDF/FTC/RPV. One patient in each arm experienced virologic failure without treatment-emergent resistance. Twenty-seven patients discontinued prematurely (8.0\% RPV vs. $4.7 \% \mathrm{EFV})$, the most frequent reasons being adverse events $(3.3 \% \mathrm{vs} .0 .5 \%$, respectively), site closure $(1.9 \%$ vs. $0.5 \%)$, loss to follow-up $(0.9 \%$ vs. $1.4 \%)$ and consent withdrawal (0.9\% vs. $1.4 \%)$.

Conclusion: In adults with suppressed viral load on first-line NNRTI-based ART in LMICs, switching to an STR of TDF/FTC/RPV was non-inferior to TDF/FTC/EFV in maintaining high rates of viral suppression with a comparable tolerability profile.

Keywords: LMIC; Single-Tablet-Regimen; Virologically suppressed adults; Treatmentemergent Resistance; SALIF.

\section{Introduction}

Current HIV treatment guidelines ${ }^{1,2,3}$ recommend that antiretroviral therapy (ART), administered as a single-tablet regimen (STR), can be initiated in all patients living with HIV, regardless of clinical stage and CD4+ cell count. In low- and middle-income countries (LMICs), there is a substantial unmet need for affordable STR options. The WHO policy brief from July $2018^{4}$ stated that dolutegravir (DTG)-based regimens may be recommended as a preferred first-line regimen for people living with HIV initiating ART, and the alternative first-line treatment regimen is efavirenz (EFV)-based. These first-line recommended treatments may result in some patients experiencing neuropsychiatric events or other tolerability issues, $5,6,7$ while the use of nevirapine (NVP) is associated with the risk of hepatotoxicity and skin reactions. ${ }^{8}$ Given that DTG is not

Corresponding author: Perry Mohammed, PMohamme@its.jnj.com

Dates: Received: 24 Jan. 2019|Accepted: 23 Mar. 2019|Published: 23 July 2019

How to cite this article: Munderi P, Were E, Avihingsanon A, et al. Switching at Low HIV-1 RNA into Fixed Dose Combinations: TDF/FTC/ RPV is non-inferior to TDF/FTC/EFV in first-line suppressed patients living with HIV. S Afr J HIV Med. 2019;20(1), a949. https://doi. org/10.4102/sajhivmed.v20i1.949

Copyright: (C 2019. The Authors. Licensee: AOSIS. This work is licensed under the Creative Commons Attribution License. 
recommended for use in non-pregnant women who are trying to conceive or during the first trimester of pregnancy because of concerns about a possible increased risk of neural tube defects, ${ }^{9}$ a viable alternative to DTG is needed. There is, thus, an unmet need for additional efficacious, well-tolerated and more affordable alternative ART regimens, particularly in LMICs. ${ }^{10}$

Rilpivirine (RPV; TMC278) is a second-generation nonnucleoside reverse transcriptase inhibitor (NNRTI) with a good safety profile ${ }^{11}$ and convenient once-daily dosing, with a reduced special effort access price of US $\$ 40$ per patient per year in sub-Saharan Africa and least developed countries, ${ }^{12}$ making it a good candidate component for an affordable STR in LMICs. ${ }^{13,14,15,16,17} \mathrm{RPV}$, in combination, is indicated in treatment-naïve patients 12 years of age and older with a viral load of HIV-1 RNA $\leq 100,000$ copies/mL. ${ }^{18}$ Tenofovir disoproxil fumarate (TDF) / lamivudine (3TC) or emtricitabine (FTC) with RPV is listed as a 'preferred' first-line regimen in European guidelines, ${ }^{3}$ has recently been adopted as a preferred first-line regimen in South African guidelines ${ }^{19}$ and is a recommended 'alternative' regimen in the United States and Thailand. ${ }^{2,20}$

Approval of RPV was based on findings from two doubleblind, placebo-controlled, phase 3 trials, ECHO and THRIVE, comparing RPV with EFV, most commonly in combination with TDF and FTC, in treatment-naive patients. ${ }^{6,7,21}$ In a 48 week pooled analysis of these trials, RPV was non-inferior to EFV both in patients with viral loads $\leq 100000$ copies $/ \mathrm{mL}$ (90\% vs. $84 \%$; $95 \%$ confidence interval [CI]: $+1.6,+11.5)$ and with viral loads $>100000$ to $\leq 500000$ copies $/ \mathrm{mL}$ (80\% vs. 83\%; 95\% CI: $-9.8,+3.7),{ }^{21}$ but non-inferiority was not achieved for patients with viral loads $>500000$ copies $/ \mathrm{mL}$ (70\% vs. $76 \%$; $95 \%$ CI: $-20.4,+8.30$ ). In addition to these pivotal trials using the individual agents, the STR of TDF/ FTC/RPV has been evaluated in both treatment-naive patients and virologically suppressed patients, including in at least one LMIC setting. In these studies, TDF/FTC/RPV was found to be non-inferior to several different ART regimens, including protease inhibitor (PI)- and NNRTIbased combinations. ${ }^{22,23,24,25}$ Hence, in Europe and the United States, TDF/FTC/RPV is indicated for use in treatment-naive patients with HIV-1 RNA $\leq 100000$ copies $/ \mathrm{mL}$ and for patients with suppressed viral load for $\geq 6$ months prior to switching therapy and without known resistance-associated mutations to NNRTIs, TDF or FTC. ${ }^{26,27}$

Preclinical studies of RPV showed no evidence of teratogenicity RPV and therefore may be an option in populations with large proportions of HIV-infected women of childbearing potential who have access to regular viral load monitoring. ${ }^{28}$ Pharmacokinetic studies of RPV in pregnancy reveal that most women achieve effective plasma concentrations of RPV. ${ }^{29,30,31}$ The Antiretroviral Pregnancy Registry showed no increased risk in birth defects after first trimester RPV exposures as of January 2018. ${ }^{32}$ Furthermore, according to both the US Department of Health and Human Services Recommendations for Use of Antiretroviral Drugs in Pregnant HIV-1-Infected Women and the 2017 European
AIDS Clinical Society guidelines, there are sufficient data from use in pregnancy to recommend RPV as an alternative agent in ART-naive pregnant women with viral loads $\leq 100000$ copies $/ \mathrm{mL}$ and CD4+ counts $>200$ cells $/ \mathrm{mm}^{3} \cdot 3,33$

This study was, therefore, designed to examine the utility of switching to the STR of TDF/FTC/RPV in LMIC patients with suppressed viral loads, who were on an NNRTI-based first-line ART.

\section{Methods}

Switching at Low HIV-1 RNA into Fixed Dose Combinations (SALIF) was a 48-week, multicentre, phase $3 \mathrm{~b}$, randomised, open-label study designed to demonstrate non-inferiority of RPV to EFV (both coformulated with TDF and FTC) in maintaining HIV-1 RNA suppression (defined as HIV-1 RNA $<400$ copies $/ \mathrm{mL}$ ) among adult patients in LMICs on firstline NNRTI-based ART (with EFV or NVP) with HIV-1 RNA $<50$ copies $/ \mathrm{mL}$. The study was conducted at 23 sites in Cameroon, Kenya, Senegal, South Africa, Uganda and Thailand from 23 August 2013 to 27 October 2015.

Ethics committee approval was obtained at all participating centres in accordance with the principles of the 2008 Declaration of Helsinki. Central randomisation was based on a computer-generated schedule prepared before the study by the sponsor. Randomisation was balanced by using randomly permuted blocks and stratified by baseline NNRTI. A prespecified interim analysis was performed once all patients had reached week 24 or discontinued earlier, and was reviewed by an independent data monitoring committee. Data from this study have been presented previously. ${ }^{34,35}$

All patients remained on study until the last patient reached the week 48 visit. Patients were then switched to an investigator-selected treatment according to local prescribing practice. In countries where a RPV-based regimen was not yet available, patients with suppressed HIV-1 RNA levels receiving TDF/FTC/RPV could continue in post-trial access programmes until RPV was publicly available in the country.

\section{Study patients}

The study included adults ( $\geq 18$ or 21 years of age, depending on national legislation of patient's country) with documented HIV-1 infection, who had been receiving first-line NNRTIbased ART (defined as two nucleoside reverse transcriptase inhibitors [NRTIs] with either EFV or NVP) for at least 1 year, and the same ART for at least 8 weeks, before screening. Previous changes in NRTI background regimen were allowed, but patients who had previously switched from EFV to NVP for toxicity reasons were not eligible. At screening, eligible patients had to have suppressed viral loads, commonly accepted to be a plasma HIV-1 RNA $<50$ copies $/ \mathrm{mL}^{36}$ a CD4+ cell count of more than 200 cells $/ \mathrm{mm}^{3}$, and a preference to change their current ART for reasons of simplification and/or NRTI toxicity. Patients also needed to have access to at least one meal a day and have a normal 
electrocardiogram (ECG) to be eligible. Patients co-infected with Mycobacterium tuberculosis, who were likely to require rifampicin-based treatment during the study, were excluded. Written informed consent was obtained from each patient prior to the screening procedures.

\section{Treatment}

At baseline, patients were randomly assigned (1:1) to receive an STR of either TDF (300 mg)/FTC (200 mg)/RPV (25 mg) or TDF (300 mg)/FTC (200 mg)/EFV (600 mg). Both products were supplied by the sponsor and given in accordance with the product labels at the recommended dose of one tablet per day. Patients randomised to TDF/FTC/RPV were advised to take the medication with food, whereas patients randomised to TDF/FTC/EFV were advised to take it on an empty stomach at bedtime. To assess adherence, patients were asked to bring the study drug containers, whether empty or not, to each study visit.

\section{Assessments}

Blood samples were collected at screening, baseline, weeks 4, $12,24,36$ and 48 , and every 24 weeks up to study end or until discontinuation and then at post-treatment follow-up. HIV-1 RNA was measured at a central laboratory, using the Abbott RealTime HIV-1 RNA assay with a lower limit of quantification of 40 copies/mL. Patients with a plasma HIV-1 RNA level $\geq 50$ copies $/ \mathrm{mL}$ were counselled on treatment adherence, and had blood samples collected for re-testing at the central laboratory at up to 8-week intervals until the plasma HIV-1 RNA was $<50$ copies/mL or the plasma HIV-1 RNA level was confirmed by two consecutive tests to be $\geq 400$ copies/ $\mathrm{mL}$. Patients with a confirmed plasma HIV-1 RNA level $\geq 400$ copies / $\mathrm{mL}$ measured at the central laboratory were classified as virologic failures. The confirmatory viral load sample was tested for genotypic drug resistance at the central laboratory.

CD4+ cell counts were determined at a central laboratory at screening, baseline, every 24 weeks up to study end or until discontinuation and then at post-treatment follow-up.

\section{Safety}

Safety monitoring (adverse events [AEs], including HIV-related events, clinical laboratory analyses, vital signs and physical examination) was performed throughout the treatment phase until study end. Electrocardiograms were recorded at screening, weeks 24 and 48, or at treatment discontinuation if earlier. The following $\mathrm{AE}$ classes of interest were investigated based on previous data from the RPV pivotal studies: rashes, neuropsychiatric events, potential QT prolongation-related events, hepatic events and endocrinological events. In addition, hyperglycaemia and new onset diabetes were analysed based on reported AEs during the study.

\section{Statistical analysis and endpoints}

The primary objective was to demonstrate non-inferiority of a TDF/FTC/RPV STR versus TDF/FTC/EFV STR in the percentage of patients with plasma HIV-1 RNA levels $<400$ copies/mL after 48 weeks (non-inferiority margin of $10 \%$ ) using a modified Food and Drug Administration (FDA) Snapshot method. ${ }^{31}$ Patients were classified as virologic responders if their HIV-1 RNA was $<400$ copies/mL within the time window of the week 48 visit (between week 42 and week 58), or if a single HIV-1 RNA value $\geq 400$ copies $/ \mathrm{mL}$ within the time window was not confirmed by a second measurement - the definition of virologic suppression selected was $<400$ copies $/ \mathrm{mL}$, to reflect the real-life practice in LMICs where a viral load of $<1000$ copies/mL should be taken as evidence as suppression. ${ }^{37}$ Patients with no HIV-1 RNA measurement within the time window of the week 48 visit were considered non-responders.

Secondary endpoints were non-inferiority in the percentage of patients with plasma HIV-1 RNA levels $<50$ copies $/ \mathrm{mL}$ after 48 weeks (modified FDA Snapshot method), rates of virologic failure during the 48 weeks of treatment with HIV-1 RNA levels $\geq 400$ or $\geq 50$ copies $/ \mathrm{mL}$ (non-virologic failure-censored analysis excluding patients who discontinued the study with HIV-1 RNA $<400$ or $<50$ copies $/ \mathrm{mL}$ ), change in CD4+ cell count, loss of treatment options, as defined by treatmentemergent drug resistance, and adherence to study treatment based on tablet count at each study visit up to week 48 .

Assuming response rates of $90 \%$ at 48 weeks for both treatment arms, 192 patients were required per arm to establish noninferiority of TDF/FTC/RPV versus TDF/FTC/EFV, with a maximum allowable difference of $10 \%$, a one-sided significance level of $2.5 \%$, and $90 \%$ power. To account for a maximum of up to $10 \%$ major protocol deviations that would result in exclusion of patients from the per protocol (PP) analysis, 213 patients were planned to be recruited in each treatment arm, resulting in 426 randomised patients in total.

The primary efficacy analysis was conducted on the intentto-treat (ITT) population (all randomised patients who had taken at least one dose of study drug, regardless of their compliance with the protocol). This analysis was repeated for the PP population (a subset of the ITT population that excluded patients with major protocol deviations). As prespecified in the statistical analysis plan (SAP), treatment arms were compared using the Cochran-Mantel-Haenszel method, adjusted for the stratification variable (use of EFV vs. NVP at the screening visit). TDF/FTC/RPV was considered non-inferior to TDF/FTC/EFV if the lower limit of the $95 \% \mathrm{CI}$ of the difference in efficacy was $\geq 10 \%$. Analysis of the percentages of patients with HIV-1 RNA levels $<50$ copies/mL, a secondary efficacy outcome, used the same statistical methods as the primary analysis.

Subgroup analyses of the virologic response were performed in the ITT population for the following pre-defined groups: NNRTI taken at screening (as stratified), baseline CD4+ count category, sex, country and treatment adherence. The ITT population was used for all safety analyses; as pre-specified in the SAP, there was no formal statistical testing of safety parameters in the study. 


\section{Ethical consideration}

Ethics committee approval was obtained at all participating centres in accordance with the principles of the 2008 Declaration of Helsinki.

\section{Results}

\section{Study patients}

Patients were recruited between 23 August 2013 and 14 August 2014. Treatment duration was between 48 and 108 weeks. Of 492 patients screened, 66 were excluded and 426 were randomised (213 in each arm); two patients in the TDF/ FTC/EFV arm did not start randomised therapy (one was randomised in error and one withdrew consent). The ITT population comprised 424 patients (Figure 1).

Overall, demographic and baseline disease characteristics were well balanced between the two treatment arms (Table 1). Most patients (309/424 [72.9\%]) were black people and of African origin (315/424 [74.3\%]), with 271/424 (63.9\%) being women, mostly of childbearing age.

All patients were taking EFV- or NVP-based regimen at screening. Most patients (415/424 [97.9\%]) were taking a non-STR before being enrolled in the study. After randomisation, in the TDF/FTC/EFV arm, 116/211 (55.0\%) patients remained on EFV and 95/211 (45.0\%) patients changed their NVP for EFV. In the TDF/FTC/RPV arm, $115 / 213(54.0 \%)$ patients were taking an EFV-based regimen and $98 / 213(46.0 \%)$ patients were taking an NVP-based regimen at screening. In the TDF/FTC/RPV arm, all (213 [100.0\%]) patients changed their NNRTI to RPV at randomisation. In addition, all but two patients (99.1\%) had a switch in both their NNRTI and NRTI, while in the TDF/ FTC/EFV arm, only $45.5 \%$ had to switch both their NNRTI and NRTI. In total, 397 (93.6\%) of 424 patients stayed on study medication until study end. Of the patients who discontinued prematurely, 17/213 (8.0\%) had received RPV and 10/211 (4.7\%) had received EFV. High adherence rates (more than 95\% adherence based on tablet count) were documented in 95.8\% (204/213) switched to TDF/FTC/RPV and in $97.6 \%(206 / 211)$ switched to TDF/FTC/EFV.

\section{Efficacy}

The primary endpoint of HIV-1 RNA $<400$ copies $/ \mathrm{mL}$ at week 48 (ITT, modified FDA Snapshot analysis) was reached by $200 / 213(93.9 \%)$ patients in the TDF/FTC/RPV arm and $203 / 211(96.2 \%)$ patients in the TDF/FTC/EFV arm with a difference of $-2.3 \%(95 \%$ CI: $-6.44,+1.84)$, demonstrating non-inferiority of TDF/FTC/RPV $(p=0.0003)$ (Figure 2$)$. In the PP population, virologic suppression was achieved by 198/207 (95.7\%) patients in the TDF/FTC/RPV arm and $200 / 207$ (96.6\%) patients in the TDF/FTC/EFV arm (difference $0.9 \%, 95 \%$ CI: $-4.66,+2.72$ ) (Figure 3). The results

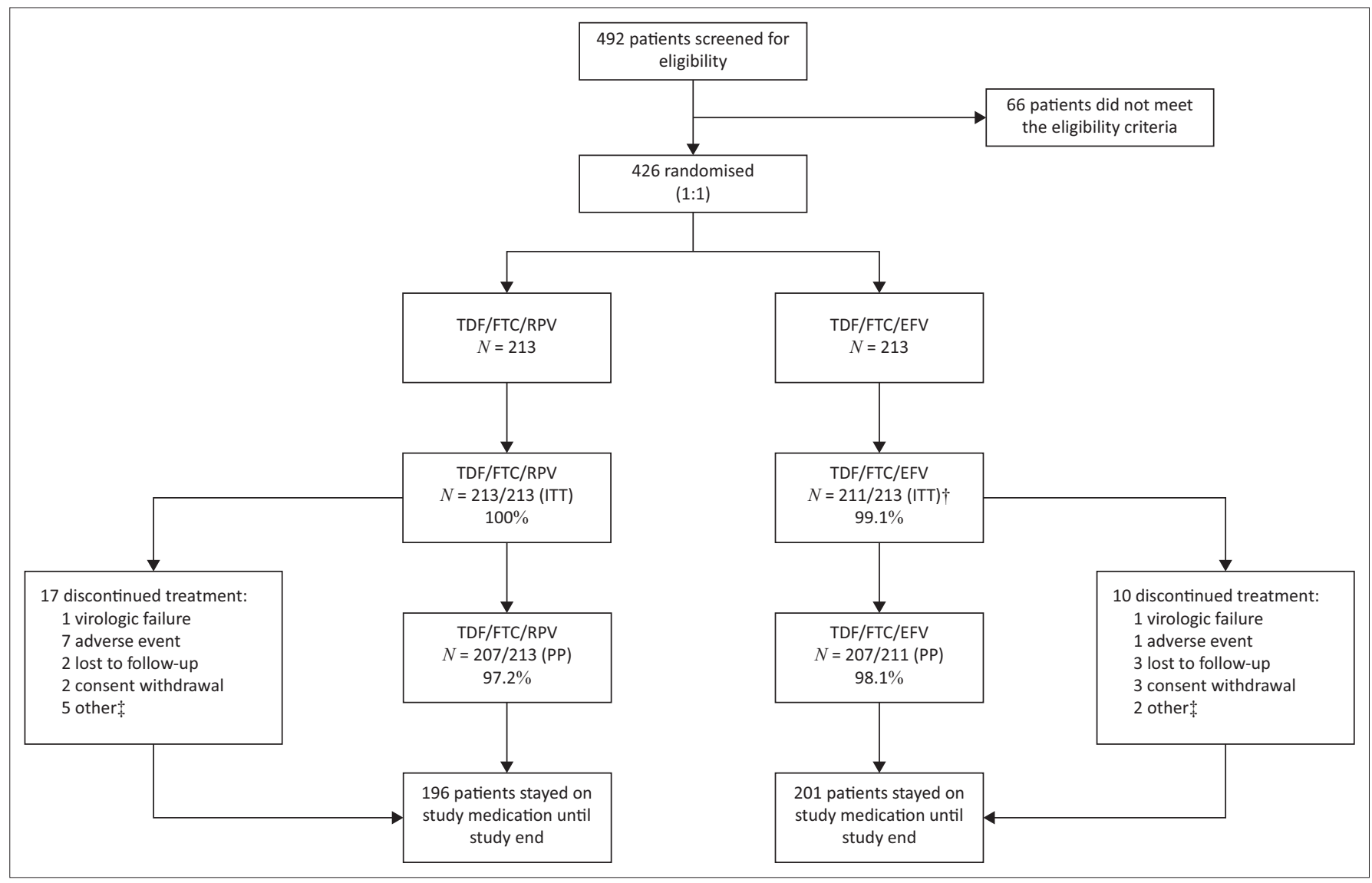

$\dagger$, One patient randomised in error; one patient withdrew consent prior to treatment. $\ddagger$, One site was closed for administrative reasons. For personal reasons, four patients in the TDF/FTC/RPV arm and one patient in the TDF/FTC/EFV arm did not consent to continuation at another site.

FIGURE 1: Patient disposition. 
TABLE 1: Baseline and disease characteristics.

\begin{tabular}{|c|c|c|c|c|c|c|c|c|c|c|c|c|}
\hline \multirow[t]{2}{*}{ Characteristic } & \multicolumn{4}{|c|}{ TDF/FTC/RPV $(n=213)$} & \multicolumn{4}{|c|}{ TDF/FTC/EFV $(n=211)$} & \multicolumn{4}{|c|}{ All patients $(n=424)$} \\
\hline & $n$ & $N$ & $\%$ & s.d. & $n$ & $N$ & $\%$ & s.d. & $n$ & $N$ & $\%$ & s.d. \\
\hline Female & 137 & - & 64.3 & - & 134 & - & 63.5 & - & 271 & - & 63.9 & - \\
\hline Women of childbearing potential & 98 & 137 & 71.5 & - & 97 & 134 & 72.4 & - & 195 & 271 & 72.0 & - \\
\hline Mean age, years & 40.6 & - & - & 8.0 & 40.6 & - & - & 8.7 & 40.6 & - & - & 8.3 \\
\hline \multicolumn{13}{|l|}{ Race } \\
\hline Black people & 157 & - & 73.7 & - & 152 & - & 72.0 & - & 309 & - & 72.9 & - \\
\hline Asian & 51 & - & 23.9 & - & 58 & - & 27.5 & - & 109 & - & 25.7 & - \\
\hline Other & 5 & - & 2.3 & - & 1 & - & 0.5 & - & 6 & - & 1.4 & - \\
\hline \multicolumn{13}{|l|}{ Nationality } \\
\hline Cameroon & 16 & - & 7.5 & - & 13 & - & 6.2 & - & 29 & - & 6.8 & - \\
\hline Kenya & 36 & - & 16.9 & - & 37 & - & 17.5 & - & 73 & - & 17.2 & - \\
\hline Senegal & 17 & - & 8.0 & - & 8 & - & 3.8 & - & 25 & - & 5.9 & - \\
\hline South Africa & 33 & - & 15.5 & - & 30 & - & 14.2 & - & 63 & - & 14.9 & - \\
\hline Thailand & 51 & - & 23.9 & - & 58 & - & 27.5 & - & 109 & - & 25.7 & - \\
\hline Mean BMI (kg/m²) & 24.25 & - & - & 4.8 & 24.11 & - & - & 5.0 & 24.18 & - & - & 4.9 \\
\hline \multicolumn{13}{|l|}{ Mode of HIV infection } \\
\hline Heterosexual contact & 188 & - & 88.3 & - & 188 & - & 89.1 & - & 376 & - & 88.7 & - \\
\hline Men having sex with men & 13 & - & 6.1 & - & 16 & - & 7.6 & - & 29 & - & 6.8 & - \\
\hline Other & 12 & - & 5.6 & - & 7 & - & 3.3 & - & 19 & - & 4.5 & - \\
\hline Mean time since diagnosis, years & 7.6 & - & - & 4.6 & 8.2 & - & - & 4.8 & 7.9 & - & - & 4.7 \\
\hline Mean time since first ART, years & 5.8 & - & - & 3.3 & 6.0 & - & - & 3.3 & 5.9 & - & - & 3.3 \\
\hline Mean CD4+ cell count, cells $/ \mathrm{mm}^{3}$ & 545.3 & - & - & 228.2 & 549.3 & - & - & 207.7 & 547.3 & - & - & 218.0 \\
\hline \multicolumn{13}{|l|}{ Hepatitis reactive } \\
\hline Hepatitis B surface antigen & 12 & - & 5.6 & - & 10 & - & 4.7 & - & 22 & - & 5.2 & - \\
\hline Hepatitis $\mathrm{C}$ antibody & 3 & - & 1.4 & - & 5 & - & 2.4 & - & 8 & - & 1.9 & - \\
\hline \multicolumn{13}{|l|}{ NNRTI at screening } \\
\hline EFV & 115 & - & 54.0 & - & 116 & - & 55.0 & - & 231 & - & 54.5 & - \\
\hline NVP & 98 & - & 46.0 & - & 95 & - & 45.0 & - & 193 & - & 45.5 & - \\
\hline $3 T C+T D F$ & 117 & - & 54.9 & - & 112 & - & 53.1 & - & 229 & - & 54.0 & - \\
\hline Other & 7 & - & 3.3 & - & 10 & - & 4.7 & - & 17 & - & 4.0 & - \\
\hline
\end{tabular}

3TC, lamivudine; ART, antiretroviral therapy; BMI, body mass index; EFV, efavirenz; FTC, emtricitabine; NNRTI, non-nucleoside reverse transcriptase inhibitor; NRTI, nucleoside reverse transcriptase inhibitor; NVP, nevirapine; RPV, rilpivirine; s.d., standard deviation; TDF, tenofovir disoproxil fumarate; ZDV, zidovudine.

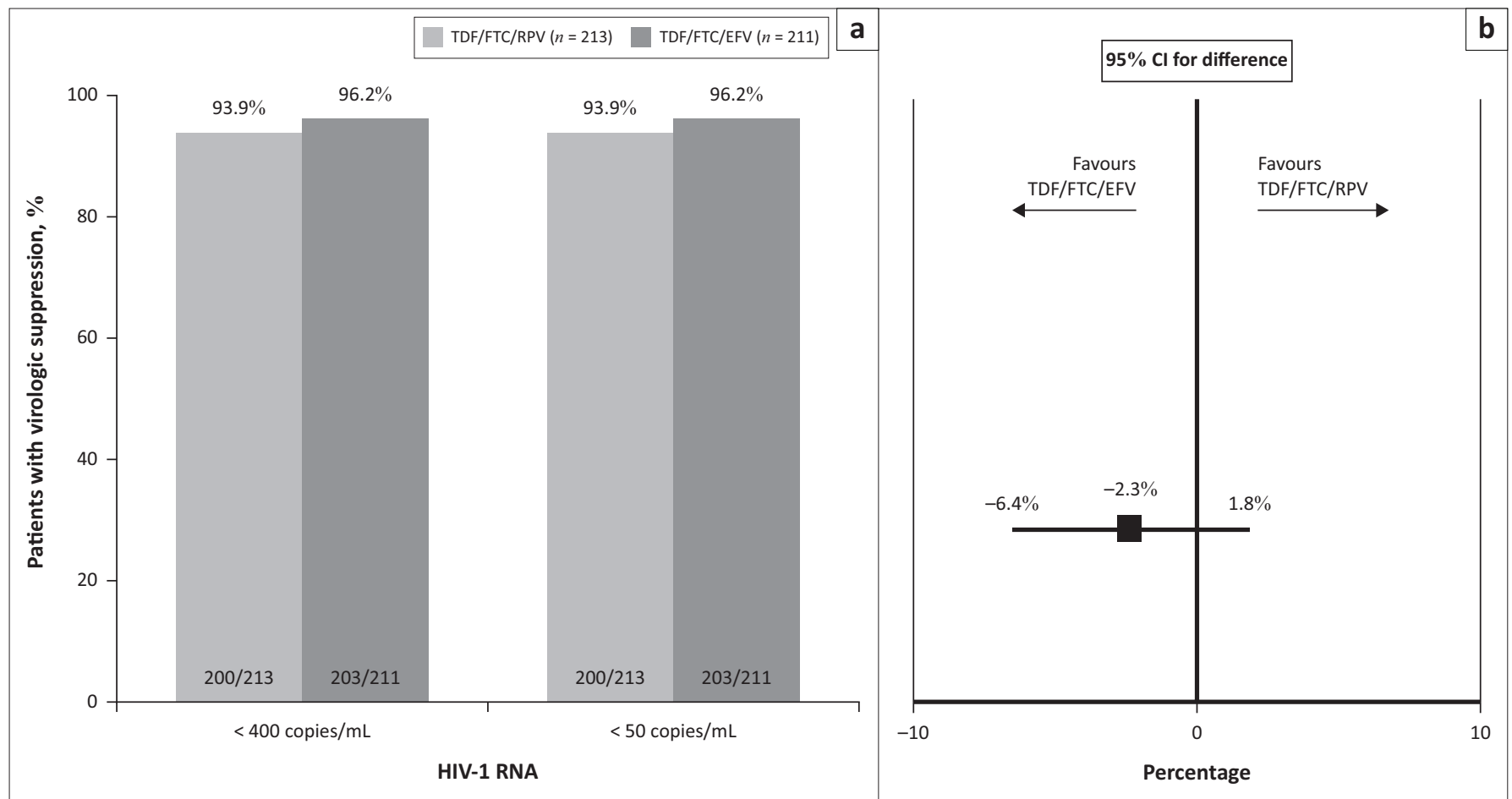

FIGURE 2: (a) Comparison of TDF/FTC/RPV and TDF/FTC/EFV showing a) $\%$ of patients with plasma HIV-1 RNA < 400 copies/mL (primary endpoint) and < 50 copies/mL at week 48 (ITT, modified FDA Snapshot analysis) and (b) difference in the primary endpoint between the two arms demonstrating non-inferiority. 
for the secondary endpoint of HIV-1 RNA $<50$ copies/mL at week 48 were identical to those for the primary endpoint for both ITT and PP populations (Figures 2 and 3).

In the ITT population, $13 / 213$ patients $(6.1 \%)$ in the TDF/ FTC/RPV arm were non-responders at week 48. Among the non-responders, five patients discontinued because of AEs, seven for other reasons and one patient experienced virologic failure. In the TDF/FTC/EFV arm, 8/211 patients (3.8\%) were non-responders at week 48 : one patient discontinued because of AEs, six for other reasons and one patient experienced virologic failure. Four patients in the TDF/FTC/ RPV arm and one patient in the TDF/FTC/EFV arm who discontinued for 'other reasons' did so because they did not

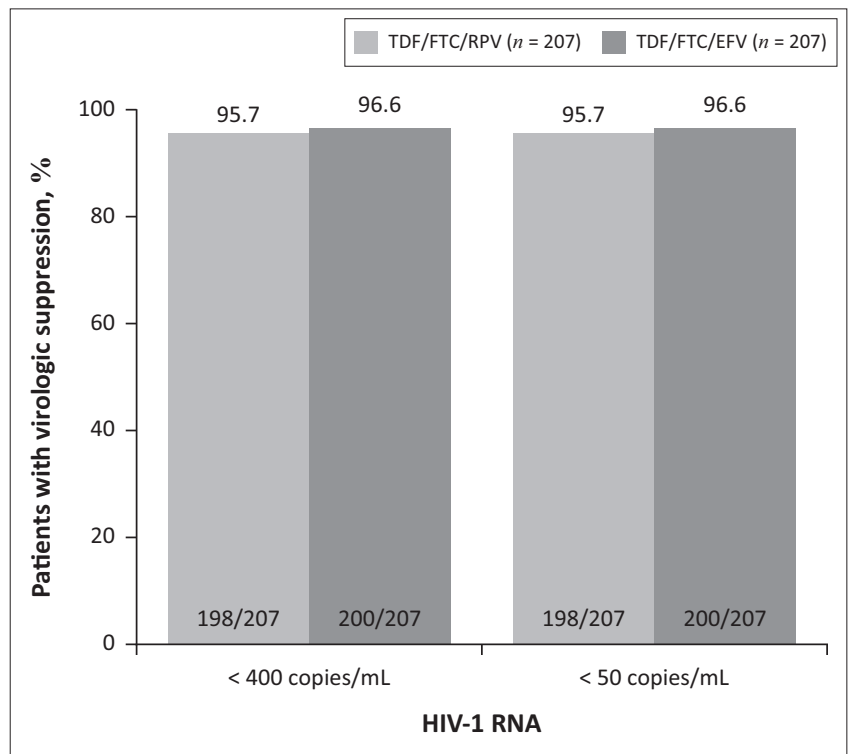

FIGURE 3: Plasma HIV-1 RNA < 400 and $<50$ copies $/ \mathrm{mL}$ at week 48 (PP population, modified FDA Snapshot). re-consent to continue the study at another site after their initial site closed because of administrative reasons. In the PP population, $9 / 207$ patients $(4.3 \%)$ and $7 / 207$ patients $(3.4 \%)$ were non-responders in the TDF/FTC/RPV and TDF/FTC/ EFV group, respectively.

The mean (s.d.) increase in CD4+ cell count from baseline at week 48 was 26.2 (125.14) cells $/ \mathrm{mm}^{3}$ in the TDF/FTC/RPV group and 6.1 (140.06) cells $/ \mathrm{mm}^{3}$ in the TDF/FTC/EFV group.

No resistance-associated mutations of the pre-defined list (IAS-USA NRTI, IAS-USA NNRTI, extended NNRTI or RPV resistance-associated mutations or primary IAS-USA PI mutations) were detected in the samples from two patients who had experienced virologic failure, thus there was no loss of treatment options observed in this study.

\section{Subgroup analyses}

For patients receiving EFV-based regimens at screening, a virologic response of HIV-1 RNA < 400 copies / $\mathrm{mL}$ at week 48 was achieved by 114/116 (98.3\%) patients who remained on EFV and $107 / 115(93.0 \%)$ of those who switched to RPV (difference $-5.2 \%$; 95\% CI: $-10.45,-0.01$ ). For patients receiving NVP-based regimens at screening, the corresponding virologic response rate was $93.7 \%(89 / 95)$ in the TDF/FTC/EFV arm and 94.9\% (93/98) in the TDF/FTC/ RPV arm (difference 1.2\%; 95\% CI: $-5.34,+7.76$ ). The study was not powered to detect non-inferiority between sub groups of the NNRTI at screening. No major differences were observed when stratifying virologic response by sex, baseline CD4+ cell count, adherence or country (Table 2).

\section{Safety}

Safety data were collected for all patients up until study end. There were no relevant differences in the incidence of AEs

TABLE 2: Virologic response (HIV-1 RNA $<400$ copies/mL) by subgroups at week 48 (ITT, modified FDA Snapshot).

\begin{tabular}{|c|c|c|c|c|c|c|c|c|}
\hline \multirow[t]{2}{*}{ Variable } & \multicolumn{3}{|c|}{ TDF/FTC/RPV } & \multicolumn{3}{|c|}{ TDF/FTC/EFV } & \multirow[t]{2}{*}{ Difference (\%) } & \multirow[t]{2}{*}{$95 \% \mathrm{Cl}$} \\
\hline & $n$ & $N$ & $\%$ & $n$ & $N$ & $\%$ & & \\
\hline \multicolumn{9}{|l|}{ NNRTI at screening } \\
\hline EFV & 107 & 115 & 93.0 & 114 & 116 & 98.3 & -5.2 & $-10.45,-0.01$ \\
\hline NVP & 93 & 98 & 94.9 & 89 & 95 & 93.7 & 1.2 & $-5.34,+7.76$ \\
\hline \multicolumn{9}{|l|}{ Baseline CD4+ } \\
\hline$<200$ cells $/ \mathrm{mm}^{3}$ & 1 & 1 & 100 & 3 & 3 & 100 & 0 & $0.00,0.00$ \\
\hline 200-349 cells $/ \mathrm{mm}^{3}$ & 39 & 41 & 95.1 & 25 & 26 & 96.2 & -1.0 & $-10.94,+8.87$ \\
\hline \multicolumn{9}{|l|}{ Sex } \\
\hline Female & 131 & 137 & 95.6 & 129 & 134 & 96.3 & -0.6 & $-5.34,+4.05$ \\
\hline Male & 69 & 76 & 90.8 & 74 & 77 & 96.1 & -5.3 & $-13.12,+2.49$ \\
\hline \multicolumn{9}{|l|}{ Country } \\
\hline All African countries & 153 & 162 & 94.4 & 147 & 153 & 96.1 & -1.6 & $-6.31,+3.05$ \\
\hline Cameroon & 16 & 16 & 100 & 13 & 13 & 100 & 0 & $0.00,0.00$ \\
\hline Kenya & 35 & 36 & 97.2 & 35 & 37 & 94.6 & 2.6 & $-6.42,+11.68$ \\
\hline Uganda & 59 & 60 & 98.3 & 64 & 65 & 98.5 & -0.1 & $-4.54,+4.28$ \\
\hline Thailand & 47 & 51 & 92.2 & 56 & 58 & 96.6 & -4.4 & $-13.14,+4.35$ \\
\hline \multicolumn{9}{|l|}{ Adherence } \\
\hline$>95 \%$ & 192 & 204 & 94.1 & 199 & 206 & 96.6 & -2.5 & $-6.55,+1.58$ \\
\hline$\leq 95 \%$ & 8 & 9 & 88.9 & 4 & 5 & 80.0 & 8.9 & $-31.74,+49.52$ \\
\hline
\end{tabular}

$\mathrm{Cl}$, confidence interval; EFV, efavirenz; FDA, Food and Drug Administration; FTC, emtricitabine; ITT, intent-to-treat; NNRTI, non-nucleoside reverse transcriptase inhibitor; NVP, nevirapine; RPV, rilpivirine; TDF, tenofovir disoproxil fumarate.

$\dagger$, One of the clinical sites in Senegal was closed before the end of the trial. This led to some patients not being followed up for the full 48 weeks and thus a lower response rate in the ITT Snapshot analysis. 
between the two arms, except for Division of AIDS (DAIDS) grade 3 or 4 AEs which were more commonly reported in patients receiving TDF/FTC/EFV compared with TDF/ FTC/RPV (56 [26.5\%] vs. 40 [18.8\%], respectively). Regardless of NNRTI at screening, the incidence of treatment-emergent DAIDS grade 3 or 4 AEs was lower in the TDF/FTC/RPV arm compared with TDF/FTC/EFV. For patients who switched from EFV at baseline, the incidence was $15.7 \%$ $(18 / 115)$ in the TDF/FTC/RPV arm versus $24.1 \%(28 / 116)$ in the TDF/FTC/EFV arm; for those patients who switched from NVP at baseline, the incidence was $22.4 \%$ (22/98) versus $29.5 \%$ (28/95), respectively.

The most frequently reported AEs at least possibly related to the study drugs were vertigo (5 [2.3\%] in the TDF/FTC/ RPV arm vs. 11 [5.2\%] in the TDF/FTC/EFV arm), headache (10 [4.7\%] vs. $6[2.8 \%])$, dizziness $(2[0.9 \%]$ vs. $8[3.8 \%])$, increased appetite $(8[3.8 \%]$ vs. $1[0.5 \%])$ and nightmares $(0$ vs. $8[3.8 \%]$ ) (Table 3$)$.

More patients in the TDF/FTC/EFV arm than in the TDF/ FTC/RPV arm experienced grade 3 or higher lipid

TABLE 3: Clinical adverse events and laboratory abnormalities.

\begin{tabular}{|c|c|c|c|c|}
\hline \multirow[t]{2}{*}{ Adverse event } & \multicolumn{2}{|c|}{ TDF/FTC/RPV $(n=213)$} & \multicolumn{2}{|c|}{ TDF/FTC/EFV $(n=211)$} \\
\hline & $n$ & $\%$ & $n$ & $\%$ \\
\hline Any adverse event & 178 & 83.6 & 174 & 82.5 \\
\hline $\begin{array}{l}\text { Drug-related adverse events } \\
\text { (all grades) }\end{array}$ & 65 & 30.5 & 53 & 25.1 \\
\hline \multicolumn{5}{|c|}{ Drug-related adverse events (all grades) in $\geq 1 \%$ of patients in either treatment arm } \\
\hline Headache & 10 & 4.7 & 6 & 2.8 \\
\hline Dizziness & 2 & 0.9 & 8 & 3.8 \\
\hline Vertigo & 5 & 2.3 & 11 & 5.2 \\
\hline Insomnia & 5 & 2.3 & 1 & 0.5 \\
\hline Nightmares & - & - & 8 & 3.8 \\
\hline Peripheral neuropathy & 4 & 1.9 & 2 & 0.9 \\
\hline Generalised pruritus & 2 & 0.9 & 3 & 1.4 \\
\hline Increased amylase & 5 & 2.3 & - & - \\
\hline Nausea & 5 & 2.3 & 2 & 0.9 \\
\hline Diarrhoea & 1 & 0.5 & 4 & 1.9 \\
\hline Increased appetite & 8 & 3.8 & 1 & 0.5 \\
\hline Renal and urinary disorders & 1 & 0.5 & 5 & 2.4 \\
\hline Grade $3-4$ adverse events & 40 & 18.8 & 56 & 26.5 \\
\hline $\begin{array}{l}\text { Drug-related grade } 3-4 \text { adverse } \\
\text { events }\end{array}$ & 13 & 6.1 & 4 & 1.9 \\
\hline \multicolumn{5}{|c|}{ Drug-related grade $3-4$ adverse events in $\geq 1 \%$ of patients in either treatment arm } \\
\hline Amylase increased & 5 & 2.3 & - & - \\
\hline $\begin{array}{l}\text { Alanine aminotransferase } \\
\text { increased }\end{array}$ & 3 & 1.4 & - & - \\
\hline Serious adverse events & 16 & 7.5 & 11 & 5.2 \\
\hline Drug-related serious adverse events & 3 & 1.4 & 1 & 0.5 \\
\hline Deaths & 1 & $0.5 \dagger$ & - & - \\
\hline $\begin{array}{l}\text { Discontinuations because of } \\
\text { adverse events }\end{array}$ & 7 & $3.3 \%$ & 1 & $0.5 \S$ \\
\hline \multicolumn{5}{|c|}{ Select grade 3-4 laboratory abnormalities } \\
\hline Amylase & 6 & 2.8 & 11 & 5.3 \\
\hline Alanine aminotransferase & 4 & 1.9 & 2 & 0.9 \\
\hline Aspartate aminotransferase & 1 & 0.5 & 1 & 0.5 \\
\hline Total cholesterol & - & - & 4 & 1.9 \\
\hline LDL cholesterol & 2 & 0.9 & 11 & 5.2 \\
\hline Triglycerides & - & - & 1 & 0.5 \\
\hline Hyperglycaemia & 3 & 1.4 & - & - \\
\hline
\end{tabular}

ALT, alanine aminotransferase; EFV, efavirenz; FTC, emtricitabine; LDL, low-density lipoprotein; RPV, rilpivirine; TDF, tenofovir disoproxil fumarate.

$t$, Myocardial infarction, unrelated to study medication; $\$$, Elevated ALT grade $3(n=2)$, Q́T prolongation grade $3(n=2)$, increased creatinine $(n=1)$, tachycardia $(n=1)$, tuberculosis $(n=1) ; \S$, Lipoatrophy. abnormalities (total cholesterol: 0 in the TDF/FTC/RPV arm vs. 4 [1.9\%], low-density lipoprotein [LDL] cholesterol: 2 [0.9\%] vs. 11 [5.2\%], triglycerides: 0 vs. 1 [0.5\%]).

Adverse events leading to discontinuation were reported in seven (3.3\%) patients switched to TDF/FTC/RPV and in one $(0.5 \%)$ patient receiving TDF/FTC/EFV. All AEs leading to permanent discontinuation were observed in at most one patient in any treatment arm, except for alanine aminotransferase increases and ECG QT prolongation, which both occurred in two $(0.9 \%)$ patients in the TDF/FTC/ RPV arm.

In the TDF/FTC/EFV group, a lower rate of treatmentemergent neuropsychiatric events of interest was seen in the subgroup of patients who were receiving EFV at screening (20/116 patients, $17.2 \%)$ compared with the rate in patients receiving NVP at screening (39/95 patients, $41.1 \%)$. This difference between the subgroups within the TDF/FTC/EFV group was seen consistently for most individual neuropsychiatric events recorded.

In the subgroup switched from NVP-based regimens, $41.1 \%$ $(39 / 95)$ of patients in the TDF/FTC/EFV arm experienced a neuropsychiatric event of interest, compared with only $30.6 \%$ (30/98) of the patients who switched to the TDF/FTC/RPV arm (Table 4).

TABLE 4: Treatment-emergent neuropsychiatric events of interest in the subgroup of patients switching from NVP and the full ITT population.

\begin{tabular}{|c|c|c|c|c|c|c|c|c|}
\hline \multirow[t]{3}{*}{ Adverse event } & \multicolumn{4}{|c|}{ TDF/FTC/RPV } & \multicolumn{4}{|c|}{ TDF/FTC/EFV } \\
\hline & \multicolumn{2}{|c|}{$\begin{array}{c}\text { NNRTI at } \\
\text { screening: } \\
\text { NVP }(n=98)\end{array}$} & \multicolumn{2}{|c|}{$\begin{array}{l}\text { All patients } \\
(n=213)\end{array}$} & \multicolumn{2}{|c|}{$\begin{array}{l}\text { NNRTI at } \\
\text { screening: NVP } \\
(n=95)\end{array}$} & \multicolumn{2}{|c|}{$\begin{array}{l}\text { All patients } \\
(n=211)\end{array}$} \\
\hline & $n$ & $\%$ & $n$ & $\%$ & $n$ & $\%$ & $n$ & $\%$ \\
\hline $\begin{array}{l}\text { Any treatment- } \\
\text { emergent } \\
\text { neuropsychiatric } \\
\text { event of interest }\end{array}$ & 30 & 30.6 & 59 & 27.7 & 39 & 41.1 & 59 & 28.0 \\
\hline \multicolumn{9}{|c|}{ Nervous system disorders } \\
\hline Headache & 17 & 17.3 & 37 & 17.4 & 16 & 16.8 & 28 & 13.3 \\
\hline Dizziness & 3 & 3.1 & 7 & 3.3 & 11 & 11.6 & 13 & 6.2 \\
\hline Somnolence & 6 & 6.1 & 11 & 5.2 & 2 & 2.1 & 2 & 0.9 \\
\hline Hypersomnia & - & - & - & - & 1 & 1.1 & 1 & 0.5 \\
\hline Head discomfort & - & - & - & - & - & - & 1 & 0.5 \\
\hline Memory impairment & - & - & - & - & - & - & 1 & 0.5 \\
\hline \multicolumn{9}{|l|}{ Psychiatric disorders } \\
\hline Nightmare & 4 & 4.1 & 4 & 1.9 & 6 & 6.3 & 9 & 4.3 \\
\hline Insomnia & 5 & 5.1 & 10 & 4.7 & 2 & 2.1 & 4 & 1.9 \\
\hline Depression & 1 & 1.0 & 1 & 0.5 & 1 & 1.1 & 2 & 0.9 \\
\hline Abnormal dreams & - & - & - & - & 1 & 1.1 & 1 & 0.5 \\
\hline Anxiety & - & - & - & - & 1 & 1.1 & 1 & 0.5 \\
\hline Libido decreased & - & - & - & - & 1 & 1.1 & 1 & 0.5 \\
\hline Libido increased & - & - & - & - & 1 & 1.1 & 2 & 0.9 \\
\hline Mood swings & - & - & - & - & 1 & 1.1 & 1 & 0.5 \\
\hline Stress & - & - & 1 & 0.5 & 1 & 1.1 & 2 & 0.9 \\
\hline Restlessness & - & - & 1 & 0.5 & - & - & - & - \\
\hline \multicolumn{9}{|c|}{ Ear and labyrinth disorders } \\
\hline Vertigo & 6 & 6.1 & 11 & 5.2 & 12 & 12.6 & 16 & 7.6 \\
\hline \multicolumn{9}{|l|}{ Eye disorders } \\
\hline Photophobia & - & - & 1 & 0.5 & 2 & 2.1 & 2 & 0.9 \\
\hline Vision blurred & 1 & 1.0 & 1 & 0.5 & 2 & 2.1 & 2 & 0.9 \\
\hline
\end{tabular}

EFV, efavirenz; FTC, emtricitabine; NVP, nevirapine; RPV, rilpivirine; TDF, tenofovir disoproxil fumarate. 


\section{Discussion}

SALIF examined the effect of switching to TDF/FTC/RPV in patients from LMICs with suppressed viral loads who were on an NNRTI-based first-line ART. This study is important because it provides additional data on the utility of TDF/FTC/RPV as a viable alternative for virologically suppressed patients on first-line NNRTI-based regimen in LMICs and in a study population that is predominantly female because women comprised $>60 \%$ of the patients enrolled.

The SALIF data add to the evidence from the SPIRIT study, which examined TDF/FTC/RPV STR as a switch option from a PI-based regimen in mostly Caucasian men in highincome settings, ${ }^{22}$ and the NEAR-RWANDA study, which demonstrated non-inferior efficacy and comparable safety of a TDF/FTC/RPV STR versus NVP-based ART in Rwanda. ${ }^{25}$ Taken together, these data support the use of RPV-based STR regimens in virologically suppressed patients. Given that viral load measurements prior to ART initiation are not routinely conducted in many LMICs, RPV-based STR regimens are an appropriate switch option for patients with suppressed viral loads. These patients have demonstrated sufficient adherence and might benefit from a switch, particularly given concerns around the safety profile of EFVand NVP-based regimens.

Recent reports from Europe have provided encouraging data on the tolerability of TDF/FTC/RPV in clinical practice, which may be transferable to the LMIC settings. ${ }^{38,39,40,41,42}$ In addition, the introduction of STRs containing tenofovir alafenamide (TAF) $25 \mathrm{mg}$ instead of TDF $300 \mathrm{mg}$, or an STR of DTG/RPV, could potentially offer increased long-term tolerability at lower dosing and, eventually, lower costs..$^{43,44,45}$ For virologically suppressed patients such as those in the SALIF trial, who are stable on ART and have already demonstrated high adherence, the risk of virologic failure is considered low. Therefore, switching to an STR may further motivate patients to stay on therapy while leading to fewer medication errors and supporting long-term adherence. This is in line with current normative guidance for mature ART programmes, which recommends differentiated models of care for patients who are stable on ART. ${ }^{46}$

Increased rates of rash and neuropsychiatric events have been reported following RPV and EFV treatment; ${ }^{21,47}$ therefore, patients in SALIF were closely monitored for these AEs. In studies in treatment-naive patients, most rash events (3\% with RPV vs. 14\% with EFV) occurred during the first 48 weeks of treatment, ${ }^{21}$ with few additional patients experiencing rashes during the second year. ${ }^{47}$ SALIF included patients who had already been successfully treated with NNRTIs, and rashes were rarely seen. EFV can cause neuropsychiatric side effects, which often resolve within the first weeks of treatment. ${ }^{10}$ This study could not confirm the improved central nervous system tolerability of RPV compared with EFV seen in treatment-naive patients. ${ }^{21}$ However, the subgroup analysis of patients who switched from NVP to either RPV or EFV showed tolerability differences in favour of RPV.

The strength of this study is that it illustrates the benefit of STRs for patients in resource-limited settings who have tolerability issues with currently available NNRTIs and who are already virologically suppressed. The main limitation of the study was that all patients entering the TDF/FTC/RPV arm changed the previous NNRTI component of their regimen, while $>50 \%$ of the patients in the TDF/FTC/EFV arm had previously received an EFV-based regimen. Switching to a new regimen may confer a potential risk for new tolerability or safety issues. Furthermore, the treatmentemergent neuropsychiatric events in the subgroup of patients switching from EFV are discordant with general tolerability data (that show that EFV is associated with higher rates of neuropsychiatric events compared with other NNRTIs, including NVP) ${ }^{48}$ This suggests that our safety findings are subject to some inherent bias of the study design. This negative bias might explain why the ITT analysis showed no differences in tolerability, while other studies in treatment-naive patients have demonstrated a generally more favourable tolerability profile of RPV compared with EFV. $6,7,21,22,24,47$ A control group of nonswitchers (or deferred switchers) staying on their original ART might help address such inherent biases in future switch studies. Another limitation of the study is the openlabel design, which may influence the reporting of side effects and discontinuation rates. For instance, QT prolongations were reported in three patients on TDF/ FTC/RPV and three patients receiving TDF/FTC/EFV; while two of the three patients receiving TDF/FTC/RPV discontinued their regimen, none in the EFV arm discontinued. A caveat to the generalisability of the study results is that trial candidates with CD4 cell counts $<200$ cells $/ \mathrm{mm}^{3}$ were excluded. Also, it should be noted that the definition of viral suppression used in the study was $<400$ copies/mL rather than $<50$ copies/mL; this was chosen to reflect real-life practice in LMICs and to account for blips, and is within the recommended WHO guidance to use a definition < 1000 copies/mL in LMICs. Finally, our study required participants, as an inclusion criterion, to have access to at least one meal a day, a situation that does not necessarily always pertain in sub-Saharan Africa and other LMIC regions.

\section{Conclusion}

In adults from LMICs with suppressed viral load on first-line NNRTI-based therapy, switching to an STR of TDF/FTC/ RPV was non-inferior to an STR of TDF/FTC/EFV in maintaining high rates of viral suppression, with comparable safety at 48 weeks. Our findings support the use of TDF/ FTC/RPV as a viable alternative to both EFV- and NVPbased regimens in LMICs, where access to a wider variety of affordable ART options is urgently needed. 


\section{Acknowledgements}

The authors would like to thank all the patients, all investigators and their teams at the following centres for their participation in SALIF: Cameroon: Pascale Abena Messomo Mbida, Cabinet Medical IDOC, Douala; Roselyne Toby, Yaoundé Central Hospital, Yaoundé. Kenya: Edwin Were, Partners in Prevention, Eldoret; Gaudensia Mutua, KAVI, Kangemi, Nairobi; Gina Ouattara, KAVI, Kenyatta Hospital, Nairobi; Lucas Otieno, Kombewa Clinical Trials Unit, Nyanza; Reena Shah, Aga Khan University, Nairobi. Senegal: Baye Moussa Samba, District Centre de Gaspard Kamara, Dakar; Kouro Bousso, Centre de Sante Domninique de Pikine, Pikine. South Africa: Lerato Mohapi, Perinatal HIV Research Unit, Chris Hani Baragwanath Hospital, Soweto; Johan Lombaard, Josha Research, Bloemfontein; Rosie Mngqibisa, Enhancing Care Foundation Wentworth Hospital, Wentworth, Durban; Karla Mellet, University of the Witwatersrand, Helen Joseph Hospital, Themba Lethu Research Centre, Johannesburg; Gita Ramjee, HIV Prevention Research Unit, Medical Research Council, Westville, KwaZulu-Natal. Uganda: Paula Munderi, MRC/UVRI Uganda Research Unit on AIDS, Entebbe; Andrew Kambugu, Infectious Diseases Institute, Mulago Hospital Complex, Kampala; Cissy Kityo, Joint Clinical Research Centre, Kampala; Fred Collins Semitala, Makerere University Joint AIDS Program, Kampala; Francis Kiweewa, Makerere University Walter Reed Project, Kampala. Thailand: Anchalee Avihingsanon, HIV-NAT - The Netherlands Australia Thailand Research Collaboration, Bangkok; Weerawat Manosuthi, Bamrasnaradura Infectious Disease Institute, Amphur Mueang Nonthaburi; Khuanchai Supparatpinyo, Ries Chiang Mai University Hospital, Chiang Mai; Winai Ratanasuwan, Siriraj Hospital Department of Infectious Disease Faculty of Medicine, Bangkok. The authors acknowledge Scientific Pathways, part of Nucleus Global, for editorial assistance, funded by Janssen Pharmaceutica NV.

\section{Competing interests}

The authors have declared that no competing interests exist.

\section{Authors' contributions}

All authors reviewed the article and endorsed the data and conclusions.

\section{Funding}

Marjolein Jansen: employee of Janssen Pharmaceutical Companies of Johnson \& Johnson and holds stock in Johnson \& Johnson. Perry Mohammed: employee of Janssen Pharmaceutical Companies of Johnson \& Johnson and holds stock in Johnson \& Johnson. Ceyhun Bicer: received consulting fees from Janssen Pharmaceutica NV; Yvon van Delft: employee of Janssen Pharmaceutical Companies of Johnson \& Johnson and holds stock in Johnson \& Johnson.

\section{Data availability statement}

Data sharing is not applicable to this article as no new data were created or analysed in this study.

\section{Disclaimer}

The views and opinions expressed in this article are those of the authors and do not necessarily reflect the official policy or position of any affiliated agency of the authors.

\section{References}

1. British HIV Association guidelines for the treatment of HIV-1-positive adults with antiretroviral therapy 2015 (2016 interim update). August 2016. [cited 2019 Apr 2]. Available from: https://www.bhiva.org/file/RVYKzFwyxpgil/treatment-guidelines2016-interim-update.pdf.

2. AIDSinfo. Panel on antiretroviral guidelines for adults and adolescents. Guidelines for the use of antiretroviral agents in HIV-1-infected adults and adolescents. 2018 [cited 2019 Apr 2] Available from: https://aidsinfo.nih.gov/contentfiles/ Ivguidelines/adultandadolescentgl.pdf.

3. European AIDS Clinical Society/EACS. EACS guidelines 9.0. October 2017 [cited 2019 Apr 2] Available from: http://www.eacsociety.org/files/guidelines_9.0-english.pdf.

4. World Health Organization. Updated recommendations on first-line and secondline antiretroviral regimens and post-exposure prophylaxis and recommendations on early infant diagnosis of HIV. July 2018. [cited 2019 Apr 2] Available from: http://apps.who.int/iris/bitstream/handle/10665/273632/WHO-CDS-HIV-18.18eng.pdf?ua $=1$.

5. Hoffmann C, Wolf E, Schewe K, et al. CNS Toxicity of dolutegravir is not associated with psychiatric conditions or higher plasma exposure. Abstract 424 , presented at: Conference on Retroviruses and Opportunistic Infections; 2018 Mar 4-7; Boston, MA. Boston, MA: CROI Foundation/IAS-USA; 2018.

6. Cohen $\mathrm{CJ}$, Andrade-Villanueva J, Clotet B, et al. Rilpivirine versus efavirenz with two background nucleoside or nucleotide reverse transcriptase inhibitors in treatmentnaive adults infected with HIV-1 (THRIVE): A phase 3, randomised, non-inferiority trial. Lancet. 2011;378(9787):229-237. https://doi.org/10.1016/S0140-6736(11)60983-5

7. Molina JM, Cahn P, Grinsztejn B, et al. Rilpivirine versus efavirenz with tenofovir and emtricitabine in treatment-naive adults infected with HIV-1 (ECHO): A phase 3 randomised double-blind active-controlled trial. Lancet. 2011;378(9787):238-246. https://doi.org/10.1016/S0140-6736(11)60936-7

8. Boehringer Ingelheim. Nevirapine PI: Nevirapine prescribing information. 2016 [cited 2019 Mar]. Available from: https://docs.boehringer-ingelheim.com/ Prescribing\%20Information/PIs/Viramune/Viramune.pdf

9. WHO. WHO Statement on DTG - Geneva 18 May 2018. Potential safety issue affecting women living with HIV using dolutegravir at the time of conception. 2018 [cited 2019 Apr 2]. Available from: https://www.who.int/medicines/ publications/drugalerts/Statement_on_DTG_18May_2018final.pdf.

10. Vitoria M, Ford N, Doherty M, Flexner C. Simplification of antiretroviral therapy: A necessary step in the public health response to HIV/AIDS in resource-limited settings. Antivir Ther. 2014;19 Suppl 3:31-37. https://doi.org/10.3851/IMP2898

11. Ford N, Lee J, Andrieux-Meyer I, Calmy A. Safety, efficacy, and pharmacokinetics of rilpivirine: Systematic review with an emphasis on resource-limited settings. HIV AIDS (Auckl). 2011;3:35-44. https://doi.org/10.2147/HIV.S14559

12. Janssen Pharmaceutica, N.V. Janssen Global Public Health: HIV medicines access \& partnerships program. 2014. [cited 2019 Apr 2]. Available from: http:// partnerships.ifpma.org/uploads/documents/138_1408024768.pdf.

13. Domingo $P$, Ribera $E$. Data on rilpivirine in treatment-naïve patients. Lessons from ECHO, THRIVE and STaR. Enferm Infecc Microbiol Clín. 2013;31 Suppl 2:20-29. https://doi.org/10.1016/S0213-005X(13)70139-3

14. Yagudina R, Kulikov A, Babiy VV. Potential long-term cost savings in treatment of naïve HIV-infected patients with rilpivirine/tenofovir/emtricitabine (single tablet regimen) in the Russian Federation. Value Health. J Int Soc Pharmacoeconomics Outcomes Res 2015;18(7):A581. https://doi.org/10.1016/j.jval.2015.09.1941

15. Neogi U, Häggblom A, Singh $K$, et al. Factors influencing the efficacy of rilpivirine in HIV-1 subtype $C$ in low- and middle-income countries. J Antimicrob Chemother. 2016;71(2):367-371. https://doi.org/10.1093/jac/dkv359

16. Gianotti N, Poli A, Nozza S, et al. Efficacy and safety in clinical practice of a rilpivirine, tenofovir and emtricitabine single-tablet regimen in virologically suppressed HIV-positive patients on stable antiretroviral therapy. J Int AIDS Soc. 2015;18:20037. https://doi.org/10.7448/IAS.18.1.20037

17. Ford N, Calmy A. Improving first-line antiretroviral therapy in resource-limited settings. Curr Opin HIV AIDS. 2010;5(1):38-47. https://doi.org/10.1097/ COH.0b013e3283339b41

18. EDURANT (rilpivirine) prescribing information. Revised 02/2018. https://www. accessdata.fda.gov/drugsatfda_docs/label/2018/202022s011lbl.pdf

19. Meintjes G, Moorhouse MA, Carmona S, et al. Adult antiretroviral therapy guidelines 2017. South Afr J HIV Med. 2017;18(1):776. https://doi.org/10.4102/ sajhivmed.v18i1.776

20. Manosuthi W, Ongwandee S, Bhakeecheep S, et al. Guidelines for antiretroviral therapy in HIV-1 infected adults and adolescents 2014, Thailand. AIDS Res Ther 2015;12:12. https://doi.org/10.1186/s12981-015-0053-z 
21. Cohen CJ, Molina JM, Cahn P, et al. Efficacy and safety of rilpivirine (TMC278) versus efavirenz at 48 weeks in treatment-naive HIV-1-infected patients: Pooled versus efavirenz at 48 weeks in treatment-naive Acquir Immune Defic Syndr. 2012;60(1):33-42. https://doi.org/10.1097/ QAl.0b013e31824d006e

22. Palella FJ Jr, Fisher M, Tebas $P$, et al. Simplification to rilpivirine/emtricitabine/ tenofovir disoproxil fumarate from ritonavir-boosted protease inhibitor tenofovir disoproxil fumarate from AIDS. 2014;28(3):335-344. https://doi.org/10.1097/QAD.0000000000000087

23. Rokx C, Verbon A, Rijnders BJ. Short communication: Lipids and cardiovascular risk after switching HIV-1 patients on nevirapine and emtricitabine/tenofovir-DF to rilpivirine/emtricitabine/tenofovir-DF. AIDS Res Hum Retroviruses. 2015;31(4): 363-367. https://doi.org/10.1089/aid.2014.0278

24. van Lunzen J, Antinori A, Cohen $\mathrm{CJ}$, et al. Rilpivirine vs. efavirenz-based singletablet regimens in treatment-naive adults: Week 96 efficacy and safety from a randomized phase 3b study. AIDS. 2016;30(2):251-259. https://doi.org/10.1097/ QAD.0000000000000911

25. Collins SE, Grant PM, Uwinkindi F, et al. A randomized switch from nevirapine-based antiretroviral therapy to single tablet rilpivirine/emtricitabine/tenofovir disoproxil fumarate in virologically suppressed human immunodeficiency virus-1-infected Rwandans. Open Forum Infect Dis. 2016;3(3):ofw141. eCollection 2016 Sep.

26. European Medicines Agency. Eviplera EPAR and SmPC. [updated 2017 Dec 19; cited 2018 Nov] Available from: http://www.ema.europa.eu/docs/en GB/document library/EPAR_-_Product_Information/human/002312/WC5001188002.pdf

27. Gilead Sciences. Complera prescribing information. 2017 [cited 2019 Apr 2]. Available from: http://www.gilead.com/ /media/Files/pdfs/medicines/hiv/complera/complera pi.ashx.

28. European Medicines Agency. Edurant EPAR and SmPC. 2018. [cited 2018 Nov] Available from: http://www.ema.europa.eu/docs/en_GB/document_library/EPAR_Product_Information/human/002264/WC500118874.pdf.

29. Tran AH, Best BM, Stek A, et al. Pharmacokinetics of rilpivirine in HIV-infected pregnant women. J Acquir Immune Defic Syndr. 2016;72(3):289-296. https://doi. org/10.1097/QAI.0000000000000968

30. Colbers A, Schalkwijk S, Konopnicki D, et al. Substantially lower rilpivirine plasma concentrations during pregnancy. Abstract 754 presented at: Conference on Retroviruses and Opportunistic Infections; 2017 Feb 13-16; Seattle, WA. Seattle, WA: CROI Foundation/IAS-USA; 2017.

31. Osiyemi O, Yasin S, Zorrilla $C$, et al. Pharmacokinetics of total and unbound Rilpivirine in HIV-1-infected pregnant women. Abstract 35 presented at: 7th International Workshop on HIV \& Women; 2017 Feb 11-12; Seattle, WA. Amsterdam: Virology Education; 2017. [cited 2019 Apr 2] Available from: http:// regist2.virology-education.com/2017/7 hivwomen/Abstractbook.pdf.

32. The Antiretroviral Pregnancy Registry. Interim Report: 1 January 1989 through 31 January 2018 [issued 2018 Jun; cited 2019 Apr 2]. Available from: http://www. apregistry.com/forms/interim report.pdf.

33. Panel on treatment of HIV-infected pregnant women and prevention of perinatal transmission. Recommendations for use of antiretroviral drugs in pregnant HIV-1infected women for maternal health and interventions to reduce perinatal HIV transmission in the United States. [cited 2019 Apr 2]. Available from: http:// aidsinfo.nih.gov/contentfiles/Ivguidelines/PerinatalGL.pdf.

34. Munderi P, Were E, Avihingsanon A, et al. SALIF trial: Switching suppressed firstline patients to tenofovir/emtricitabine/rilpivirine (TDF/FTC/RPV) is non-inferio to TDF/FTC/efavirenz (TDF/FTC/EFV) and could be an alternative treatment option in LMICs. Abstract THAB0104 presented at: AIDS; 2016 Jul 18-22; Durban South Africa. New York: Wiley; 2016 [cited 2018 Apr 2] Available from https:// onlinelibrary.wiley.com/doi/epdf/10.7448/IAS.19.6.21264.
35. Shah R, Ramjee G, Kityo C, et al. Safety and efficacy of TDF/FTC/RPV and TDF/FTC/ EFV - 48 week subgroup-analyses from the SALIF study. Abstract 112 presented at: 11th INTEREST Workshop; 2017 May 16-19; Lilongwe, Malawi. Reviews in at: 11th INTEREST Workshop; 2017 May 16-19; Lilongwe, Malawi. Reviews in Antiviral Therapy \& Infectious
Development (AIGHD); 2017.

36. Günthard HF, Saag MS, Benson CA, et al. Antiretroviral drugs for treatment and prevention of HIV infection in adults: 2016 recommendations of the International Antiviral Society-USA Panel. JAMA. 2016;316(2):191-210. https://doi.org/10.1001/ jama.2016.8900

37. McMahon JH, Elliott JH, Bertagnolio S, Kubiak R, Jordan MR. Viral suppression after 12 months of antiretroviral therapy in low- and middle-income countries: A systematic review. Bull World Health Organ. 2013;91(5):377-385E. https://doi. systematic review. Bull Wor
org/10.2471/BLT.12.112946

38. Pinnetti C, Di Giambenedetto S, Maggiolo F, et al. Switching to coformulated rilpivirine/emtricitabine/tenofovir in virologically suppressed patients: Data from rilpivirine/emtricitabine/tenofovir in virologically suppressed patients: Data from https://doi.org/10.1097/QAl.0000000000000727

39. Lewis JM, Smith C, Torkington A, et al. Real-world persistence with antiretroviral therapy for HIV in the United Kingdom: A multicentre retrospective cohort study. $\mathrm{J}$ Infect. 2017;74(4):401-407. https://doi.org/10.1016/j.jinf.2017.01.012

40. Häggblom $A$, Lindbäck $S$, Gisslén $M$, et al. HIV drug therapy duration; a Swedish real world nationwide cohort study on InfCareHIV 2009-2014. PLoS One. 2017;12(2):e0171227. https://doi.org/10.1371/journal.pone.0171227

41. Gianotti N, Maggiolo F, Cozzi-Lepri A, et al. Durability of RPV- and INSTI-based 1stline regimens in HIV-infected patients starting ART with a $V L<100000$ copies $/ \mathrm{mL}$ : Data from the ICONA Foundation Study. Abstract MOPEB0300. Presented at: IAS; 2017 Jul 23-26; Paris, France. New York: Wiley; 2017.

42. Sculier D, Gayet-Ageron A, Battegay M, et al. Rilpivirine use in the Swiss HIV cohort study: A prospective cohort study. BMC Infect Dis. 2017;17(1):476. https://doi. org/10.1186/s12879-017-2579-2

43. Raffi F, Orkin C, Clarke A, et al. Brief report: Long-term (96-week) efficacy and safety after switching from tenofovir disoproxil fumarate to tenofovir alafenamide in HIV-infected, virologically suppressed adults. J Acquir Immune Defic Syndr. 2017;75(2):226-231. https://doi.org/10.1097/QAI. 0000000000001344

44. Walensky RP, Horn TH, Paltiel AD. The epi-TAF for tenofovir disoproxil fumarate? Clin Infect Dis. 2016;62(7):915-918. https://doi.org/10.1093/cid/ civ1000

45. Llibre JM, Hung CC, Brinson C, et al. Phase III SWORD 1\&2: Switch to DTG+RPV maintains virologic suppression through 48 wks. Abstract 44LB. Presented at: Conference on Retroviruses and Opportunistic Infections; 2017 Feb 13-16; Seattle, WA. Seattle, WA: CROI Foundation/IAS-USA; 2017.

46. World Health Organization. Consolidated guidelines on the use of antiretrovira drugs for treating and preventing HIV infection. Recommendations for a public health approach. Second edition 9 June 2016. [cited 2019 Apr 2] Available from: http://apps.who.int/iris/bitstream/10665/208825/1/9789241549684_ eng.pdf.

47. Cohen CJ, Molina JM, Cassetti I, et al. Week 96 efficacy and safety of rilpivirine in treatment-naive, HIV-1 patients in two Phase III randomized trials. AIDS 2013;27(6):939-950. https://doi.org/10.1097/QAD.0b013e32835cee6e

48. Ford N, Shubber Z, Pozniak A, et al. Comparative safety and neuropsychiatric adverse events associated with efavirenz use in first-line antiretroviral therapy: A systematic review and meta-analysis of randomized trials. Acquir Immune Defic Syndr. 2015;69(4):422-429. https://doi.org/10.1097/ QAI.0000000000000606 